\title{
Die Schweizerische Gesellschaft für Pulmonale Hypertonie SGPH
}

John-David Aubert

Prof. Dr. med.

Präsident der SGPH

\begin{abstract}
Von einer Pulmonalen Hypertonie ist dann die Rede, wenn der mittlere pulmonal-arterielle Druck in Ruhe grösser als $25 \mathrm{mmHg}$ ist, oder der rechtsventrikuläre systolische Druck über $40 \mathrm{mmHg}$ ansteigt. Eine idiopathische Pulmonale Hypertonie tritt sehr selten auf (ca. 1-2 Fälle pro Million Personen), während die Prävalenz für sekundäre Formen des Lungenhochdrucks infolge anderer pulmonaler oder kardialer Organerkrankungen viel höher ist. Da die Krankheitssymptome einer Pulmonalen Hypertonie sehr uncharakteristisch sind und oft mit anderen Lungen- oder Herzleiden verwechselt werden, erfolgt die richtige Diagnose häufig relativ spät, und trotz erheblicher Fortschritte in der Diagnostik und Behandlung führt die schwere Erkrankung unbehandelt nicht selten innert weniger Jahre zum Tod.
\end{abstract}

\section{Die Aufmerksamkeit für diese lebensbedrohliche Erkrankung sollte erhöht werden}

Korrespondenz:

Administrative Geschäftsstelle der SGPH

Gabriela Deutsch c/o IMK Institut für Medizin und Kommunikation AG Münsterberg 1 CH-4001 Basel Tel. 0612713551 Fax 0612713338 ssph@imk.ch
Um die Aufmerksamkeit für diese lebensbedrohliche Erkrankung zu erhöhen und die Forschung in diesem Gebiet zu intensivieren, hat 1998 in Bern eine Gruppe von Pneumologen, Intensivmedizinern, Angiologen, Kardiologen, Pädiatern und Internisten die Schweizerische Arbeitsgruppe für Pulmonale Hypertonie SAPH gegründet. Dem Expertengremium unter der Präsidentschaft von PD Dr. Guido Domenighetti schlossen sich im Laufe der Jahre viele weitere an der Pulmonalen Hypertonie interessierte Spezialisten an und bildeten so eine fächerübergreifende Gesellschaft, die sich mit Workshops, Kongressen und sogar eigenen Studien der Erkrankung widmen.

Aufgrund der rasanten Entwicklung der Arbeitsgruppe wurde daraus im Jahr 2005 die Schweizerische Gesellschaft für Pulmonale Hypertonie SGPH gegründet, die der Ärzteschaft und den Patienten als Referenzzentrum zur Verfügung steht und folgende Ziele verfolgt:

- Information der Ärzteschaft und der Bevölkerung über die Pulmonale Hypertonie

- Erstellung und laufende Aktualisierung eines klinischen Registers mit relevanten Informationen zu den in der Schweiz behandelten Patienten

- Sicherstellung der Qualität der Behandlung von Patienten

- Förderung von Forschungsarbeiten auf dem Gebiet der Pulmonalen Hypertonie
- Regelmässige Treffen und Förderung des wissenschaftlichen Austauschs unter Kollegen

- Unterstützung von Patientenorganisationen

Der SGPH gehören aktuell 51 Mitglieder an, die sich teilweise in verschiedenen Gruppen organisieren.

Die Registry-Gruppe befasst sich mit der Erstellung und Führung eines Registers der PH-Fälle in der Schweiz. In der Datenbank werden alle relevanten Informationen über die Patienten und den Krankheitsverlauf eingetragen und verwaltet. Sie sollen danach als Grundlage für nationale Auswertungen und Vergleiche dienen. Anhand der erfassten Daten der Registry-Gruppe konnten auch bereits erste Publikationen veröffentlicht werden.

Die SGPH verfügt zudem über eine eigene Webseite (www.sgph.ch) in den drei Landessprachen, die sich sowohl an Ärzte wie auch an Patienten richtet und von der Homepage-Gruppe regelmässig aktualisiert wird. Die Webseite ist momentan in einem Aktualisierungsprozess und wurde eigens an einem zweitägigen Workshop im Mai 2010 durch verschiedene Gruppen analysiert und auf die Aktualität hin überprüft. Die Aufschaltung der aktualisierten Texte ist für den Herbst 2010 vorgesehen.

Für die Organisation von Fortbildungsmassnahmen und Kongressen ist die wissenschaftliche Kommission, bestehend aus mindestens einem ordentlichen Mitglied pro Sprachregion und dem amtierenden Präsidenten, verantwortlich. In der Regel findet alternierend jährlich mindestens ein Treffen in Form eines Workshops oder Kongresses statt. Zusätzliche Arbeitstreffen zu bestimmten Themen werden dazwischen eingestreut. Vom 8. bis 10. September 2011 organisiert die SGPH in Interlaken eine gemeinsame Jahrestagung mit folgenden Gesellschaften und Interessengemeinschaften: Intensivmedizin (SGI), Klinische Ernährung (GESKES), Notfall- und Rettungsmedizin (SGNOR) und Intensivpflege (IGIP).

Seit 2004 gibt die SGPH durchschnittlich zwei Mal pro Jahr einen Newsletter in gedruckter Form heraus. Damit verfolgt sie das Ziel, das Zielpublikum, also Allgemeinpraktiker mit Interesse an der Pulmonalen Hypertonie und Spezialisten wie Kardiologen, Pneumologen, Rheumatologen oder Intensivmediziner, für diese seltene Krankheit zu sensibilisieren und auf dem neusten Stand der Forschung zu halten. Neu wird in jeder Ausgabe auch ein aktueller Patientenfall aus einem PAH-Zentrum porträtiert.

Weitere Informationen zur SGPH erhalten Sie auf der Webseite unter www.sgph.ch oder bei der administrativen Geschäftsstelle. 